\title{
Newton-raphson method to solve systems of non-linear equations in VANET performance optimization
}

\author{
Siti Asilah Yah', Naimah Yaakob², Mohamed Elshaikh Elobaid ${ }^{3}$, Ong Bi Lynn ${ }^{4}$, R. Badlishah ${ }^{5}$, Wan \\ Aida Nadia Wan Abdullah ${ }^{6}$ \\ 1, 2, 3, 4, ${ }^{6}$ ENAC, School of Computer and Communication Engineering (SCCE), University Malaysia Perlis (UniMAP), \\ 02600 Arau, Perlis, Malaysia \\ ${ }^{5}$ Faculty of Informatics and Computing, University Sultan Zainal Abidin (UniSZA), Kuala Terengganu, Malaysia
}

\section{Article Info}

Article history:

Received Oct 24, 2018

Revised Nov 30, 2018

Accepted Dec 28, 2018

\section{Keywords:}

MAC protocol's parameters

Newton-Raphson

Non-linear Equation

VANET

\begin{abstract}
Nowadays, Vehicular Ad-Hoc Network (VANET) has got more attention from the researchers. The researchers have studied numerous topics of VANET, such as the routing protocols of VANET and the MAC protocols of VANET. The aim of their works is to improve the network performance of VANET, either in terms of energy consumption or packet delivery ratio (PDR) and delay. For this research paper, the main goal is to find the coefficient of $a, b$ and $c$ of three non-linear equations by using a NewtonRaphson method. Those three non-linear equations are derived from a different value of Medium Access Control (MAC) protocol's parameters. After that, those three coefficient is then will be used in optimization of the VANET in terms of energy, PDR, and delay.
\end{abstract}

Copyright $@ 2019$ Institute of Advanced Engineering and Science. All rights reserved.

\section{Corresponding Author:}

Siti Asilah Yah,

ENAC, School of Computer and Communication Engineering (SCCE),

University Malaysia Perlis (UniMAP),

02600 Arau, Perlis, Malaysia.

Email: ilahyah_92@yahoo.com

\section{INTRODUCTION}

The Newton-Raphson method was originally developed by Newton for solving nonlinear equations. The method at first was known as Newton method. Then, the method later refined by Raphson, and hence the method is also known as Newton-Raphson method in the literature of numerical analysis [1,2]. The method requires both the first- and second-order derivatives of $f(\lambda)$.

Newton-Raphson was selected to be used for this experiment because of its capability in solving nonlinear equations. The aim of this experiment is to find the coefficient of $a, b$ and $c$ of three different equations. After that, this three coefficient is then will be used in the optimization of the network.

The experiment was run for three different configuration which is for a low, medium and high value of MAC protocol's parameters. From the experiment, the result for three type of evaluation metrics are recorded, which are energy, delay, and packet delivery ratio (PDR). Each configuration and recorded result is then used to extract three different equation. Further explanation of this process will be discussed in section VI.

The next subsection will discuss the selection of MAC protocol's parameters for this experiment. Then, the explanation about the simulation method of this experiment is described in section III which is the methodology section. After that, the result of this experiment are clarified and newton-raphson method is discussed. Then, the conclusion of this experiment is defined. 


\section{SELECTION OF MAC PROTOCOL'S PARAMETERS}

Three MAC protocol's parameters have been decided to be taken into consideration in this research project. The three parameters which are transmission power, bitrate, and contention window are chosen because of their great impact on energy consumption and network performance of VANET. The explanation about on how these three parameters affected the network performance will be discussed as follows.

In general, the transmission power is the amount of power that is assigned to the transmitter for the data transmission process. It is known that the energy consumed by the network is determined by the uses of the transmission power. The transmission power affects the power consumption in the transmitter if it is too high which will reduce the battery lifespan and increase the interference between nodes operating at the same frequency. However, if the transmission power is too low, the signal-to-noise ratio at the receiver will be affected. The maximum transmission power for VANET is $1584.89 \mathrm{~mW}$ which is equal to $1000 \mathrm{~m}$ coverage area or transmission range [3, 4]. For this experiment, 3 value were selected as shown in Table 1 . The selected value represented the low, medium and high value of the transmission power. For this experiment, the decided values of transmission power are 500, 1000 and $1584.89 \mathrm{~mW}$, where the difference between the vectors is constant which is around $500 \mathrm{~mW}$.

The next chosen MAC protocol's parameter is bitrate. Bitrate is the amount of data (in bit) that being transmitted in per unit of time. The support of sending data at 6,12 and $24 \mathrm{Mbit} / \mathrm{s}$ are mandatory while 9, 18, 36, 48 and 54Mbit/s are optional data rates [5]. Bit rate for IEEE 802.11p has been reduce to half from previous version of IEEE 802.11 , which is from $54 \mathrm{Mb} / \mathrm{s}$ to $27 \mathrm{Mb} / \mathrm{s}$. This is due to the limitation of PHY layer regarding one of the characteristic of vehicular network which is mobility [5]. The IEEE 802.11p offers data exchange among vehicles (V2V) and between vehicles and roadside infrastructure (V2I) within a range of $1 \mathrm{~km}$ using a transmission rate of $3 \mathrm{Mbps}$ to $27 \mathrm{Mbps}$ and a vehicle velocity up to $260 \mathrm{~km} / \mathrm{h}$ [6-7].

Lastly, the contention window $(\mathrm{CW})$. The contention window is defined as a period of time in which the network is operating in contention mode. Generally, it seems that keeping $\mathrm{CW}$ size larger provide higher throughput especially in heavy load for lower priority traffics and also provide better fairness among priority classes in high loads and prevent from starvation in low priority traffics. However, to gain lower jitter in a heavy load for higher priority traffics, reducing CW size to smaller values acts better [8]. The probability that two nodes will try to access the medium at the same time is small, but when there are e.g. 100 nodes the probability that two nodes will choose the same time increase. Increasing the $\mathrm{CW}$ might be a way to deal with this but making the $\mathrm{CW}$ too large will increase delay. A solution may lie in adapting the $\mathrm{CW}$ in response to the number of nodes. If there are many nodes, CW could increase and if there are fewer nodes CW could decrease [9]. The optimal CW does not depend only on the number of active stations but also depend on the packet size [10]. When the number of competing nodes increase, it will be shown to be ineffective since the new collisions can potentially occur and cause significant performance degradation. So even the number of nodes has increased to a very large value, the nodes will use the same initial CW. As a result, a lot of collisions occur and the throughput is deteriorated. If we decrease CWmin to a value less than the optimal value, there will be more collisions, which will degrade the performance. At the same way, if we increase CWMin to a value greater than the optimal value, the packet transmitted will suffer from a longer delay, which will also degrade the performance.

The summary of that selected parameter can be referred in Table 1. From Table 1, V1 represent the vector for the low value and V2 denote the vector for the medium value of the three parameters. In addition, vector 3 (V3) in the table shows the high value of the selected parameters.

Table 1. Summarization value of the selected MAC protocol's parameters

\begin{tabular}{cccc}
\hline Vector & Transmission Power (Tx) & Contention Window (CW) & Bitrate (Mbps) \\
\hline V1 & $500 \mathrm{~mW}$ & 31 & 6 \\
V2 & $1000 \mathrm{~mW}$ & 128 & 12 \\
V3 & $1584.89 \mathrm{~mW}$ & 255 & 24 \\
\hline
\end{tabular}

\section{METHODOLOGY}

This project used OMNeT++ discrete event simulator framework (version 5.1.1) to run the simulation. Each simulation was run for 200 s and repeated for ten times in order to get the accurate result. After that, the average result for this repeated simulation was taken and tabulated. Then the result was used to calculate the Objective Function (OF) for this project as explained in section V.

For this experiment, GPSR is used for the routing protocol. GPSR is pioneer location-based routing protocol for VANET. GPSR is proposed by [9] for VANET in 2000. GPSR utilizes the position information provided by a location assist system to calculate a route to the destination. GPSR works in two modes greedy and forwarding modes. GPSR uses only one-hop neighbors' location information to find a path, it's also 
make its forwarding decision dynamically to cope with different network situations (when greedy forwarding fails to use perimeter forwarding [11-13].

In addition, the IEEE 802.11p MAC protocol has been used as the MAC protocol. The IEEE 802.11p has been proposed as a draft amendment to IEEE 802.11 to support traffic exchange in vehicular networks [14]. It makes use of the PHY supplement 802.11a and the MAC layer QoS amendment from 802.11e. Vehicular Ad-hoc Network is accomplished by adopting only a few predominant technologies employed in the wireless networking application.

For the transport protocol, the User Datagram Protocol (UDP) has been used. This is because it can be very fast, with low delay and are not affected by congestion on a connection basis. UDP has comparatively fast speed and is thus used for games or applications that require fast transmission of data [15]. Since VANET is a real-time application and the message need to be transmitted fast, UDP is chosen as the transport protocol.

Table 2 summarizes the simulation environment and parameters used in this simulation. The parameters are taken to portray a realistic vehicular traffic for an urban scenario. While Figure 1 shows the configuration in the Omnet++ simulator for the different MAC protocol's parameters.

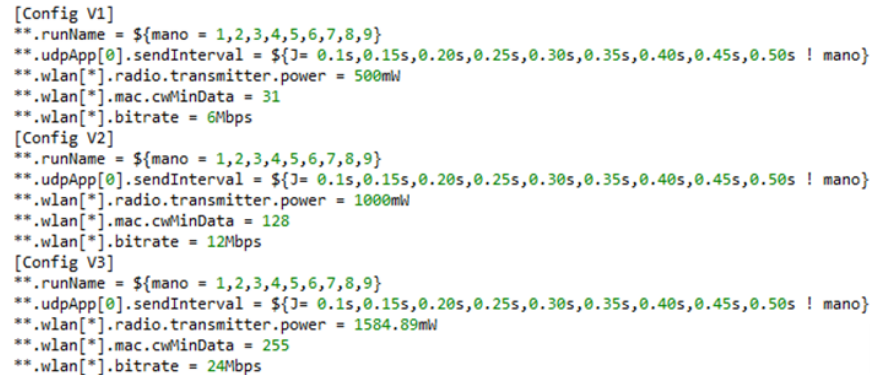

Figure 1. Omnet++ configuration for three different value of parameters

Table 2. List of parameters value

\begin{tabular}{cc}
\hline Parameters Names & Parameter Value \\
\hline Playground & $1000 \mathrm{~m}^{*} 1000 \mathrm{~m}$ \\
Number of RSU & 8 \\
Number of Vehicles & 30 \\
Speed of vehicles & $70-90 \mathrm{~km} / \mathrm{h}$ \\
Simulation Time & $200 \mathrm{~s}$ \\
Routing Protocol & GPSR \\
MAC Protocol & IEEE $802.11 \mathrm{p}$ \\
Transport Protocol & UDP \\
Mobility Type & vehicles: Linear, Rectangular \\
& RSU: Static \\
\hline
\end{tabular}

\section{EVALUATION MATRICS}

The network performance for this experiments are evaluated based on different types of simulation. Each simulation was repeated ten times to get the accurate results. Then, the average result value of the repeated simulation are calculated and tabulated to evaluate and analyse the network performance. The evaluation metrics which are selected to analyse the network performance are energy consumption, PDR, and transmission time delay. The evaluation metrics are explained in the Table 3.

Table 3. Explanation of evaluation metrics

\begin{tabular}{ll}
\hline \multicolumn{1}{c}{ Name } & \multicolumn{1}{c}{ Explanation } \\
\hline Energy Consumption & $\begin{array}{l}\text { Energy used by the node to transmit the data. The lower the energy consumption is the better since it can } \\
\text { make the sensor life time much longer. } \\
\text { Represents the number of packets that successfully received by the receiver to the number of packets sent by } \\
\text { the source. } \\
\text { PDR }=\frac{\text { Received Packets }}{\text { Sent Packets }} \\
\text { Packet Delivery Ratio } \\
\text { (PDR) }\end{array}$ \\
$\begin{array}{l}\text { The higher the value of PDR is the better since it indicate the rate of successful received data. That is means } \\
\text { less packet are drop due to collision or corrupted. } \\
\text { Transmission Time }\end{array}$ & Represents the arrival time needed by the packet to its destination. The lower the delay is the better. \\
\hline
\end{tabular}




\section{THE OBJECTIVE FUNCTION (OF)}

The aims of this work are to produce a network that can minimize the energy consumption which can make the sensor lifetime much longer and at the same time give a good network performance. To achieve these aims, the network requires a high value of packet delivery ratio (PDR), and also a low value of transmission time delay and energy consumption. If the network can achieve this value that means the network was in a good performance. So, for this work, an objective function (OF) equation as shown in 1 was used to reach those goals.

Therefore, in order to get the desired value for this project, the OF value in (1) must be low. The finding OF values is then used to optimize the system for this project. Why does the OF values need to be low? This is because, OF is directly proportional to delay and energy, and inversely proportional to PDR. Therefore, in order to get a high value of PDR and at the same time, a low value of delay and energy, the OF value must be low.

$$
f(P D R, \text { delay, Energy })=\frac{\text { Energy } * \text { Delay }}{P D R}=O F
$$

For further explanation, PDR is the rate of a successful packet transmitted. The higher the value of PDR, the higher the number of the packets that successfully transmitted by the sender to the receiver. Which means, less number of packet are loss due to collision or corrupted. In addition, PDR and throughput are directly proportional. When PDR is high, the value of throughput also high. Throughput is the average rate of successful packet that can be delivering to its destination over a communication channel per unit of time. Hence, when the value of PDR is high, the value of throughput also high which leads to the good performance of network. Therefore, the system desire a high value of PDR in order to get a good network performance. So, the value of PDR is inversed and then multiplied with other value which is delay and energy as shown in (1).

Delay is the time required by the network to complete the message transmission. Delay is measure in second per bits, which means how many second is needed to complete the transmission of one bit of data. So, from the 1 , considering that the aim of OF is to reduce or minimize the delay of the network. Hence, the value of delay is directly proportional to OF.

Lastly, energy consumption. Energy consumption is the amount of energy or power used by the network to complete the data transmission. It is measure in joule $(\mathrm{J})$. The desire value for energy is low, in order to make the sensor life time much longer. So, because of that the desire value for energy is low, hence, the value of energy are directly proportional to $\mathrm{OF}$.

In conclusion, by minimizing the value of OF, it can maximize the value of PDR and can minimize the value of delay and energy. The calculated value of OF is then used to calculate the value of coefficient a, b, and c by using Newton-Raphson method as shown in (2). Further explanation about Newton-Method will be discussed in the next section.

$$
a^{2} x_{1}+b^{2} x_{2}+c^{2} x_{3}=\frac{\text { Energy } * \text { Delay }}{P D R}=O F
$$

From (2), $x_{1}$ represent the contention window while $x_{2}$ is bitrate and $x_{3}$ is transmission range which is the MAC protocol's parameters that has been chosen to be taken into consideration in this work.

\section{RESULT AND DISCUSSION}

Table 4, 5 and 6 shows the result of the OF for the different configuration of the experiment. Table 4 shows the result for the configuration of low values of parameters, while Table 5 shows the result for the configuration of medium values of parameters. The results for the set of high values of parameters are shown in Table 6.

The experiments were run for five different sent intervals as shown in the table. One over the sent interval (1/SI) indicates the packet rate, which means how many packets are sent per second. Then, the value of OF for these five different sent interval are calculated. The average OF value for these five sent intervals is then will be used in Newton-Raphson method. 
Table 4. Result for low value of MAC protocol's parameters

\begin{tabular}{clcll}
\hline Sent interval & PDR & Energy & Delay & OF \\
\hline $0.10 \mathrm{~s}$ & 0.856857 & 13.708 & 0.48676031 & 7.7871896 \\
$0.15 \mathrm{~s}$ & 0.831305 & 12.238 & 0.72000784 & 10.599552 \\
$0.20 \mathrm{~s}$ & 0.779007 & 13.414 & 0.32328739 & 5.5668016 \\
$0.25 \mathrm{~s}$ & 0.787532 & 14.1 & 0.34533627 & 6.1829087 \\
$0.30 \mathrm{~s}$ & 0.700091 & 14.394 & 0.72886478 & 14.985604 \\
& & & Average OF & 9.0244111 \\
\hline
\end{tabular}

Table 5. Result for medium value of MAC protocol's parameters

\begin{tabular}{ccccc}
\hline Sent interval & PDR & Energy & Delay & OF \\
\hline $0.10 \mathrm{~s}$ & 0.858683 & 13.512 & 0.25394767 & 3.9960516 \\
$0.15 \mathrm{~s}$ & 0.793034 & 14.296 & 0.40717697 & 7.3401625 \\
$0.20 \mathrm{~s}$ & 0.806543 & 13.316 & 0.22489114 & 3.7129435 \\
$0.25 \mathrm{~s}$ & 0.803348 & 13.806 & 0.21192254 & 3.6420125 \\
$0.30 \mathrm{~s}$ & 0.655201 & 12.924 & 0.41553631 & 8.1965564 \\
& & & Average OF & 5.3775453 \\
\hline
\end{tabular}

Table 6. Result for high value of MAC protocol's parameters

\begin{tabular}{ccccc}
\hline Sent interval & PDR & Energy & Delay & OF \\
\hline $0.10 \mathrm{~s}$ & 0.851257 & 13.414 & 0.17150583 & 2.7025657 \\
$0.15 \mathrm{~s}$ & 0.766937 & 12.924 & 0.30303854 & 5.1066401 \\
$0.20 \mathrm{~s}$ & 0.814642 & 13.61 & 0.16634936 & 2.7791529 \\
$0.25 \mathrm{~s}$ & 0.807475 & 13.806 & 0.16820963 & 2.8760058 \\
$0.30 \mathrm{~s}$ & 0.642558 & 12.434 & 0.3182114 & 6.1576395 \\
& & & Average OF & 3.9244008 \\
\hline
\end{tabular}

From the table, it revealed that the OF value becomes smaller when the parameter's value becomes bigger. This is because when the parameter's value becomes high, the energy consumption and delay also will be low and the PDR will be high. Since the denominator (PDR) is high, the value of OF is low. This is because the OF value is inversely proportional to the denominator.

\subsection{Newton-Raphson (NR) Method for solving non-linear equations}

The NR method is used to find the coefficient $a, b$, and $c$ for 2 . To find the coefficient, three different equations are required. The three equations represent the low, medium and high value of MAC protocol's parameters configuration. For this experiments, the coefficient values are calculated based on the NR method by using Microsoft Excel.

$$
\begin{aligned}
& O F_{1}=a^{2}(31)+b^{2}(6)+c^{2}(0.5) \\
& O F_{2}=a^{2}(128)+b^{2}(12)+c^{2}(1) \\
& O F_{3}=a^{2}(255)+b^{2}(24)+c^{2}(1.58489)
\end{aligned}
$$

where; $x_{1}=$ Contention Window $(\mathrm{CW})$

$$
\begin{aligned}
& x_{2}=\text { Bitrate } \\
& x_{3}=\text { Transmission Power }
\end{aligned}
$$

As shown in (3), (4) and (5) is the equation that derived from the different set of MAC protocol's parameters value. As shown in (3) is the derivation equation for a set of low value of parameters while as shown in 4 is the equation for a set of medium value of parameters. Then the as shown in (5) is derived from the high-value set of parameters. In addition, $\mathrm{OF}_{1}, \mathrm{OF}_{2}$, and $\mathrm{OF}_{3}$ are the average objective function as calculated in section VI.

A Newton-Raphson method for solving the system of nonlinear equations requires the evaluation of a matrix, known as the Jacobian of the system, which is defined as below. 


$$
\mathbf{J}=\left[\begin{array}{ccc}
\frac{\partial O F_{1}}{\partial a} & \frac{\partial O F_{1}}{\partial b} & \frac{\partial O F_{1}}{\partial c} \\
\frac{\partial O F_{2}}{\partial a} & \frac{\partial O F_{2}}{\partial b} & \frac{\partial O F_{2}}{\partial c} \\
\frac{\partial O F_{3}}{\partial a} & \frac{\partial O F_{3}}{\partial b} & \frac{\partial O F_{3}}{\partial c}
\end{array}\right]=\left[\begin{array}{ccc}
62 a & 12 b & c \\
256 a & 24 b & 2 c \\
510 a & 48 b & 3.168 c
\end{array}\right]
$$

Then, as shown in 6 is used to calculate the coefficient of $\mathrm{a}, \mathrm{b}$, and $\mathrm{c}$. Where $\mathrm{k}$ is the current solution and $\mathrm{k}+1$ is the next solution values sequentially. To solve this equation; three predefined values for a, b, c and OF are required and will be acquired from the as shown in (3), (4) and (5). These predefined parameters will be applied to yields the set of as shown in 6 and solve it by iteration.

$$
\left[\begin{array}{l}
a_{k+1} \\
b_{k+1} \\
c_{k+1}
\end{array}\right]=\left[\begin{array}{l}
a_{k} \\
b_{k} \\
c_{k}
\end{array}\right]-\left[\begin{array}{ccc}
\frac{\partial O F_{1}}{\partial a} & \frac{\partial O F_{1}}{\partial b} & \frac{\partial O F_{1}}{\partial c} \\
\frac{\partial O F_{2}}{\partial a} & \frac{\partial O F_{2}}{\partial b} & \frac{\partial O F_{2}}{\partial b} \\
\frac{\partial O F_{3}}{\partial a} & \frac{\partial O F_{3}}{\partial b} & \frac{\partial O F_{3}}{\partial c}
\end{array}\right]^{-1} \times\left[\begin{array}{l}
O F_{1} \\
O F_{2} \\
O F_{3}
\end{array}\right]
$$

The predefined value for $\mathrm{a}, \mathrm{b}$ and $\mathrm{c}$ are 1 and the predefined value of $\mathrm{OF}$ is the average OF value from section VI. These values are then used to calculate a new set of OF as shown below.

$$
\begin{aligned}
& (31)\left(1^{2}\right)+(6)\left(1^{2}\right)+(0.5)\left(1^{2}\right)-9.0244111=28.47559=F_{1} \\
& (128)\left(1^{2}\right)+(12)\left(1^{2}\right)+(1)\left(1^{2}\right)-5.377545=135.6225=F_{2} \\
& (255)\left(1^{2}\right)+(24)\left(1^{2}\right)+(1.58489)\left(1^{2}\right)-3.9244008=276.6596=F_{3}
\end{aligned}
$$

Then, all the new calculated value of OF are applied in as shown in (6) to find the next solution values as follows.

$$
\left[\begin{array}{l}
a_{k+1} \\
b_{k+1} \\
c_{k+1}
\end{array}\right]=\left[\begin{array}{l}
1 \\
1 \\
1
\end{array}\right]-\left[\begin{array}{ccc}
62(1) & 12(1) & 1 \\
256(1) & 24(1) & 2(1) \\
510(1) & 48(1) & 3.168(1)
\end{array}\right]^{-1} \times\left[\begin{array}{l}
28.47559 \\
135.6225 \\
276.6596
\end{array}\right]
$$

This step will be repeated until the satisfied value is found. As shown in (7) below shows how to find the Jacobian transpose matrix $\left(\mathrm{J}^{-}\right)$.

$$
\mathbf{J}^{-}=\left[\begin{array}{lll}
\frac{\partial O F_{1}}{\partial a} & \frac{\partial O F_{1}}{\partial b} & \frac{\partial O F_{1}}{\partial c} \\
\frac{\partial O F_{2}}{\partial a} & \frac{\partial O F_{2}}{\partial b} & \frac{\partial O F_{2}}{\partial b} \\
\frac{\partial O F_{3}}{\partial a} & \frac{\partial O F_{3}}{\partial b} & \frac{\partial O F_{3}}{\partial c}
\end{array}\right]^{-1}=\frac{1}{\operatorname{det}(J)} \times \operatorname{Adj}(J)
$$

Table 7 shows the calculated result for coefficient $a, b$, and $c$ for four cycles. The calculated value for these three coefficients is then will be used in the optimization method. The value will be selected based on their impact on OF. For the next step, which is in the optimization step, the OF value once again will be calculated by using these three calculated coefficients, and which cycle that gives the lowest value of OF to the network will be selected to be used in optimization method.

Table 7. Result of coefficient a, b, and c for different cycle

\begin{tabular}{cccc}
\hline Cycle & a & Result & \\
& $\mathrm{b}$ & $\mathrm{c}$ \\
\hline 1st & -30.15216794 & -626.954 & 319.9396 \\
2nd & -1125.524818 & -75167.92269 & 38028.27378 \\
3rd & -453703.2039 & -8732487.719 & 4499070.324 \\
4th & -14320992.82 & -1042294910 & 527041376.7 \\
\hline
\end{tabular}




\section{CONCLUSION}

In conclusion, the main goal of this experiment was achieved which is to calculate the coefficient value of $\mathrm{a}, \mathrm{b}$, and $\mathrm{c}$. For the next step, this research work will continue with the optimization method by using the calculated coefficient from this experiments.

\section{ACKNOWLEDGEMENTS}

The research reported in this paper is supported by Research Acculturation Grant Scheme (RAGS) [Grant number: 9018-00082]. The author would also like to express gratitude to the Malaysia Ministry of Higher Education (MOHE) and University Malaysia Perlis for the facilities provided.

\section{REFERENCES EV}

[1] C. Remani, "Numerical Methods for Solving Systems of Nonlinear Equations," vol. 4301, 2013.

[2] S. Sivaganesan and M. Chandrasekaran, "Impact of various compression ratio on the compression ignition engine with diesel and mahua biodiesel," Int. J. ChemTech Res., vol. 9, no. 11, pp. 63-70, 2016.

[3] F. P. Rezha, T. S. Siadari, and Soo Young Shin, "Adaptive transmission power in cluster-based routing VANET," 2012 18th Asia-Pacific Conf. Commun., pp. 539-543, 2012.

[4] D. B. Rawat, D. C. Popescu, G. Yan and S. Olariu, "Enhancing VANET Performance by Joint Adaptation of Transmission Power and Contention Window Size," in IEEE Transactions on Parallel and Distributed Systems, vol. 22, no. 9, pp. 1528-1535, Sept. 2011

[5] A. M. S. Abdelgader and W. Lenan, "The Physical Layer of the IEEE 802. 11p WAVE Communication Standard: The Specifications and Challenges," Proc. World Congr. Eng. Comput. Sci., vol. II, pp. 22-24, 2014.

[6] Y. (Jeff) Li, “An Overview of the DSRC/WAVE Technology," in Quality, Reliability, Security and Robustness in Heterogeneous Networks, 2012, pp. 544-558.

[7] F. A. Teixeira, V. F. e Silva, J. L. Leoni, D. F. Macedo, and J. M. S. Nogueira, "Vehicular networks using the IEEE 802.11p standard: An experimental analysis," Veh. Commun., vol. 1, no. 2, pp. 91-96, 2014.

[8] A. Khalaj, N. Yazdani, and M. Rahgozar, "Effect of the contention window size on performance and fairness of the IEEE 802.11 standard,” Wirel. Pers. Commun., vol. 43, no. 4, pp. 1267-1278, 2007.

[9] R. Reinders, M. van Eenennaam, G. Karagiannis and G. Heijenk, "Contention window analysis for beaconing in VANETs," 2011 7th International Wireless Communications and Mobile Computing Conference, Istanbul, 2011, pp. 1481-1487.

[10] Elwathig Elhag, Mohamed Othman, "Adaptive Contention Window Scheme for WLANs," Int. Arab J. Inf. Technol., vol. 4, no. 4, p. 313, 2007.

[11] S. Boussoufa-Lahlah, F. Semchedine, and L. Bouallouche-Medjkoune, "Geographic routing protocols for Vehicular Ad hoc NETworks (VANETs): A survey,” Veh. Commun., vol. 11, pp. 20-31, 2018.

[12] Y. Khaled, "Routing in vehicular ad hoc networks," vol. 2018, pp. 1-2, 2008.

[13] C. A. T. H. Tee and A. C. R. Lee, "Survey of Position Based Routing for Inter Vehicle Communication System," First Int. Conf. Distrib. Framew. Appl., pp. 174-182, 2008.

[14] M. Amadeo, C. Campolo, and A. Molinaro, "Enhancing IEEE 802.11p/WAVE to provide infotainment applications in VANETs," Ad Hoc Networks, vol. 10, no. 2, pp. 253-269, 2012.

[15] K. Dalal, P. Chaudhary, and D. P. Dahiya, "Performance Evaluation of TCP and UDP Protocols in VANET Scenarios using NCTUns-6.0 Simulation Tool,” Int. J. Comput. Appl., vol. 36, no. 6, pp. 6-9, 2011. 